\title{
Estructura de comercialización de bovinos destinados al abasto de carne en la región del Papaloapan, Veracruz, México
}

\section{Commercialisation structure of cattle destined for meat supply in the region of Papaloapan, Veracruz, Mexico}

\author{
JULIO VilaboA-ARRONIZ \\ Pablo Díaz-Rivera \\ Diego Esteban Platas-Rosado \\ Octavio Ruiz-Rosado* \\ Sergio Segundo GonZÁlez-Muñoz** \\ FRANCISCO JUÁREZ-LAGUNES ***
}

\begin{abstract}
The aim of this study is to identify the distribution channels in the commercialisation of cattle destined for meat supply in the region of Papaloapan, Veracruz, as well as the function characterisation of the agents that participate in the process. Five information sources have been used and the work was carried out in seven municipalities where the greatest cattle inventory is located in the region. We calculated the central trend, dispersion, frequency and contingency tables among the different agents.
\end{abstract}

Keywords: meet supply, cattle commercialisation, meat consumption preferences.

\section{Resumen}

Los objetivos de esta investigación son identificar los canales de distribución y caracterizar la función de los agentes participantes en la comercialización de bovinos destinados al abasto de carne en la región del Papaloapan, Veracruz. Se utilizaron cinco fuentes de información y se trabajó en siete municipios donde está el mayor inventario bovino de la región. Se calcularon medidas de tendencia central, dispersión, frecuencias y tablas de contingencia entre agentes.

Palabras clave: abasto de carne, comercialización de bovino, preferencias en el consumo de carne.

* Colegio de Posgraduados, Campus Veracruz, México. Correos-e: juliovilaboa@ hotmail.com; pablod@colpos.mx; dplatas@colpos.mx; octavior@colpos.mx.

** Colegio de Posgraduados, Campus Montecillo, México. Correo-e: ssgonzal@ yahoo.com.

*** Universidad Veracruzana, México. Correo-e: juarez_lagunes@yahoo.com.mx. 


\section{Introducción}

De acuerdo con el Plan Estatal de Desarrollo (2005-2010), geográficamente el estado de Veracruz se divide en siete regiones: Huasteca, Montañas, Selva, Sotavento, Tuxtlas, Capital y Papaloapan. Esta última tiene 21 municipios y es la tercera región más extensa del estado, allí se concentra $17.8 \%$ del inventario bovino estatal; por su actividad ganadera, destacan los municipios de Playa Vicente, Tierra Blanca y Alvarado (Sagarpa-SIAP, 2005; Gobierno del Estado de Veracruz, 2005: 18). Esta región presenta ventajas comparativas que pueden posicionar a la industria pecuaria en un nivel competitivo en los ámbitos nacional e internacional; sin embargo, existen ciertos obstáculos estructurales que se deben superar para lograrlo.

Es importante identificar la estructura de comercialización de bovinos destinados al abasto de carne en la región, ya que permite tener una aproximación de la situación actual para tomar decisiones tanto individuales como de política pública que apuntalen a la industria ganadera, pues $24 \%$ de la comercialización de bovinos en el estado $(455,122)$ se realiza en la región del Papaloapan (Sedarpa, 2007), y poco más de la mitad de este porcentaje se hace mediante el esquema de comercialización no integrado (tradicional). Por ello es útil conocer los agentes que integran dicho esquema e identificar sus características, así como las razas y el tipo de bovino comercializado y, por ende, el tipo de carne que consume la población en la zona. Los objetivos de este trabajo son identificar los canales de distribución y mercados destino por tipo de bovino, así como caracterizar a los agentes participantes y su función en el proceso de mercadeo; además, se determinó el volumen de ganado bovino comercializado y se analizó a los consumidores de este tipo de carne, con la finalidad de conocer las características y limitantes de cada agente participante en el mercado.

En México, y particularmente en la región del Papaloapan, se ha subestimado la importancia de los bovinos destinados al abasto de carne para el mercado local, además, se carece de un estudio completo e información documentada para esta región que permita identificar los componentes (agentes) que lo integran, datos que se consideran importantes para planificar y desarrollar la comercialización de los productos y subproductos bovinos en su conjunto, justificado en la necesidad de mejorar la pro- 
ductividad y competitividad de los componentes que la integran (Gomes, 1999: 7).

Por esta razón, este estudio se centró en el esquema de comercialización tradicional en la región del Papaloapan, debido a que tal esquema se ha ignorado en una zona con importante actividad ganadera, considerando que cuenta con un inventario de 830,894 cabezas.

\section{Materiales y métodos}

La región del Papaloapan se encuentra entre los $17^{\circ} 50^{\prime}$ y $19^{\circ} 38^{\prime}$ $\mathrm{N}$ y entre $95^{\circ} 49^{\prime}$ y $96^{\circ} 21^{\prime} \mathrm{O}$. Esta región, la tercera más extensa $\left(10,941.96 \mathrm{~km}^{2}\right)$ de Veracruz, se ubica al suroeste del estado y colinda con las regiones de las Montañas, Sotavento y Los Tuxtlas. En la región del Papaloapan hay 21 municipios, de los cuales se seleccionaron Alvarado, Azueta, Cosamaloapan, Ignacio de la Llave, Ixmatlahuacan, Playa Vicente y Tlacotalpan, porque cuentan con $38.9 \%$ de los ganaderos y $63.3 \%$ del inventario bovino de la región.

Los agentes de la estructura de comercialización de bovinos destinados al abasto de carne considerados en este estudio son: productores primarios (ganaderos), introductores, rastros y mataderos municipales, tablajeros y consumidores. Para caracterizar la estructura de comercialización se diseñaron cinco instrumentos de encuesta para aplicarlas a cada componente.

Para determinar el tamaño de la muestra, se consideró como variable base el número promedio de bovinos por productor (830,894 bovinos y 10,196 productores), tomando como fuente el padrón de ganaderos de las asociaciones ganaderas locales (AGL) de cada municipio de la región y los datos del INEGI (2005). Para el estudio se calculó una muestra de 187 productores y las entrevistas se hicieron en las AGL de cada municipio. Se identificaron dos rastros y cinco mataderos municipales (uno por municipio) en función de la normatividad de la Dirección General de Ganadería (DGG) del gobierno del estado, así como el volumen de matanza de los años 2005 y 2006. Por las entrevistas realizadas a los administradores de los rastros y mataderos municipales, se identificaron 27 introductores de ganado bovino en la región. Para determinar el tipo de bovino sacrificado se entrevistó a los administradores de los rastros y mataderos municipales, quienes realizan el control diario de la matanza y pasan dicha información a las mesas ganaderas de cada municipio, quienes llevan el 
control mensual y reportan a la DGG, encargada de elaborar el reporte anual. Dicho reporte (2005-2006) sólo indica el sexo (hembras y machos), pero no especifica el tipo de ganado, información que fue proporcionada por los administradores de los rastros. Para los tablajeros se tomó como base el padrón de cada municipio (313 en la región) y se obtuvo un tamaño de muestra de 75 tablajeros (24.3\%); se localizaron carnicerías y expendios de carne dentro y fuera de los mercados de las cabeceras municipales, así como en localidades secundarias. Para los consumidores, se consideró la población de los municipios objeto de estudio, que representa $38.8 \%$ de la población total regional $(517,100$ habitantes), de ello obtuvimos un tamaño de muestra de 278 consumidores, que fueron entrevistados a la salida de las carnicerías y expendios de carne. Las variables analizadas por tipo de agente participante se presentan en el cuadro 1.

\section{Cuadro 1}

Variables analizadas por agente participante en el esquema de comercialización tradicional de bovinos para el abasto de carne en la región del Papaloapan

\begin{tabular}{|c|c|c|c|c|c|}
\hline $\begin{array}{l}\text { Variablel } \\
\text { agente }\end{array}$ & Productor & Introductor & $\begin{array}{c}\text { Rastro } \\
\text { municipal }\end{array}$ & Tablajero & Consumidor \\
\hline Género & $\mathrm{x}$ & $\mathrm{x}$ & & $\mathrm{x}$ & $\mathrm{x}$ \\
\hline Edad & $\mathrm{x}$ & $\mathrm{x}$ & & $\mathrm{x}$ & $\mathrm{x}$ \\
\hline Escolaridad & $\mathrm{x}$ & $\mathrm{x}$ & & $\mathrm{x}$ & $\mathrm{x}$ \\
\hline $\begin{array}{l}\text { Antigüedad en la } \\
\text { actividad }\end{array}$ & $\mathrm{X}$ & $\mathrm{X}$ & $\mathrm{X}$ & $X$ & \\
\hline $\begin{array}{l}\text { Nivel de asociación } \\
\text { Dependencia } \\
\text { económica }\end{array}$ & $\mathrm{x}$ & $\mathrm{x}$ & & $\mathrm{x}$ & \\
\hline $\begin{array}{l}\text { de la actividad } \\
\text { Preferencia o }\end{array}$ & $\mathrm{x}$ & & & $\mathrm{x}$ & $\mathrm{x}$ \\
\hline gusto por la actividad & $\mathrm{x}$ & $\mathrm{x}$ & & $\mathrm{x}$ & \\
\hline Razas utilizadas & $\mathrm{x}$ & $\mathrm{x}$ & $\mathrm{x}$ & $\mathrm{x}$ & \\
\hline $\begin{array}{l}\text { Tipo de bovino } \\
\text { destinado al abasto }\end{array}$ & $\mathrm{X}$ & $\mathrm{X}$ & & $\mathrm{x}$ & \\
\hline $\begin{array}{l}\text { Origen del bovino o } \\
\text { canal de carne (lugar) }\end{array}$ & & $\mathrm{X}$ & $\mathrm{x}$ & $\mathrm{X}$ & \\
\hline $\begin{array}{l}\text { Destino del bovino o } \\
\text { canal de carne (lugar) }\end{array}$ & r) & $\mathrm{X}$ & $\mathrm{x}$ & $\mathrm{X}$ & \\
\hline $\begin{array}{l}\text { Tipo de establecimien } \\
\text { (rastro o matadero) } \\
\text { Tipo de bovino } \\
\text { sacrificado }\end{array}$ & nto & & $\mathrm{x}$ & & \\
\hline
\end{tabular}


continúa...

\begin{tabular}{|c|c|c|c|}
\hline Productor & $\begin{array}{cc}\text { Introductor } & \text { Rastro } \\
\text { Municipal }\end{array}$ & Tablajero & Consumidor \\
\hline Porcentaje de bovino & & & \\
\hline sacrificado & $\mathrm{X}$ & & \\
\hline Nivel de operación & $\mathrm{x}$ & & \\
\hline Volumen de matanza & $\mathrm{x}$ & & \\
\hline Número de introductores & & $\mathrm{x}$ & \\
\hline Clientes principales & & $\mathrm{x}$ & \\
\hline Número de integrantes & & & \\
\hline de la familia & & & $\mathrm{x}$ \\
\hline Preferencia o gusto por & & & \\
\hline la carne bovina & & & $\mathrm{x}$ \\
\hline
\end{tabular}

$\mathrm{x}$ : variable analizada

Fuente: Elaboración propia.

Las entrevistas se realizaron durante el segundo semestre del año 2007 y utilizamos un método de muestreo no probabilístico por cuotas; el criterio de selección fue ser agente participante (productor, introductor, rastro municipal, tablajero, consumidor) en el esquema de comercialización no integrado con disponibilidad para participar en la entrevista. Los datos obtenidos se registraron en una matriz estructurada en una hoja de cálculo (Excel, 2003) para generar una base de datos por cada agente. Los datos se analizaron con el paquete estadístico SAS (2003) para calcular frecuencias, medidas de tendencia central, dispersión y tablas de contingencia para identificar los canales de distribución y caracterizar a los agentes participantes y su función en la comercialización de bovinos.

\section{Resultados y discusión}

De acuerdo con la teoría económica de libre mercado, el productor maximiza su utilidad con las restricciones de su tecnología, mientras que el consumidor maximiza su satisfacción con las restricciones de su presupuesto; ambos no tienen influencia sobre los precios del mercado, por lo que toman los precios y todo lo que se produce se consume (Tansini, 2003; Bernanke y Frank, 2007). Según Caldentey (1986: 20), el agente de comercialización es cualquier individuo o persona jurídica que, directa $\mathrm{o}$ indirectamente, añade utilidad al producto.

En México, la cadena de carne bovina es muy compleja, ya que se compite con empresas que llegan a los consumidores finales mediante cadenas comerciales de autoservicio y grandes importadores (Márquez et al., 2004: 122); además, la mayor parte 
de la producción nacional se enfoca al abasto interno (Ruiz et al., 2004: 8). En Veracruz, como en resto del país, existen dos esquemas de comercialización de bovinos: 1) el integrado a los rastros tipo inspección federal (TIF) y 2) el tradicional (no integrado) (Bravo et al., 2002: 256). En este estudio se caracterizó la estructura de comercialización de bovinos del esquema tradicional, en el cual participan los agentes productor, acopiador, introductor, rastro municipal, mayorista, tablajero y consumidor (Meléndez, 2001: 239; Bravo et al., 2002: 261; Trueta, 2003: 72); donde la participación de éstos es esencial para su funcionamiento (Bravo et al., 2002: 261), ya que presenta una escasa integración (Márquez et al., 2004: 122) que es una limitante para la competitividad de la industria, pues encarece el producto al consumidor.

Las principales actividades que realizan los agentes en este esquema de comercialización son acopio, manipulación, transporte, sacrificio, distribución y consumo (Meléndez, 2001: 243; Trueta, 2003: 68). El acopio, realizado por el introductor, consiste en recolectar bovinos (de rancho en rancho) destinados para el abasto, agruparlos en lotes e introducirlos al rastro municipal para su sacrificio. La manipulación se refiere a las operaciones de manejo y movimiento de los bovinos desde los ranchos ganaderos hasta el consumidor final. El transporte es el traslado del bovino en pie para su sacrificio en los establecimientos destinados para tal fin. El sacrificio se realiza en el rastro o matadero municipal; éste se considera como un agente indirecto, ya que sólo proporciona los servicios de sacrificio y maquila, pero no interviene en los precios del mercado. La distribución, realizada por el introductor o el tablajero, consiste en el envío de la carne en canal del rastro a las carnicerías, donde el consumidor adquiere la carne al menudeo (cortes populares) (Meléndez, 2001: 246; García-Mata et al., 2003: 152; Ruiz et al., 2004: 8; Huerta, 2005: 661).

El análisis de la información acerca de los agentes participantes en el proceso de mercadeo, permitió identificar y rediseñar la estructura de comercialización de bovinos destinados al abasto de carne, además de diferenciar el esquema de comercialización tradicional del integrado (figura 1).

Se encontraron cinco canales de distribución, aunque ésta no se hace de manera lineal como lo mencionan Meléndez (2001) y Bravo et al. (2002), sino que existen interacciones entre los agentes que conforman la estructura de comercialización. Cada uno de los agentes participantes requiere de infraestructura, insu- 


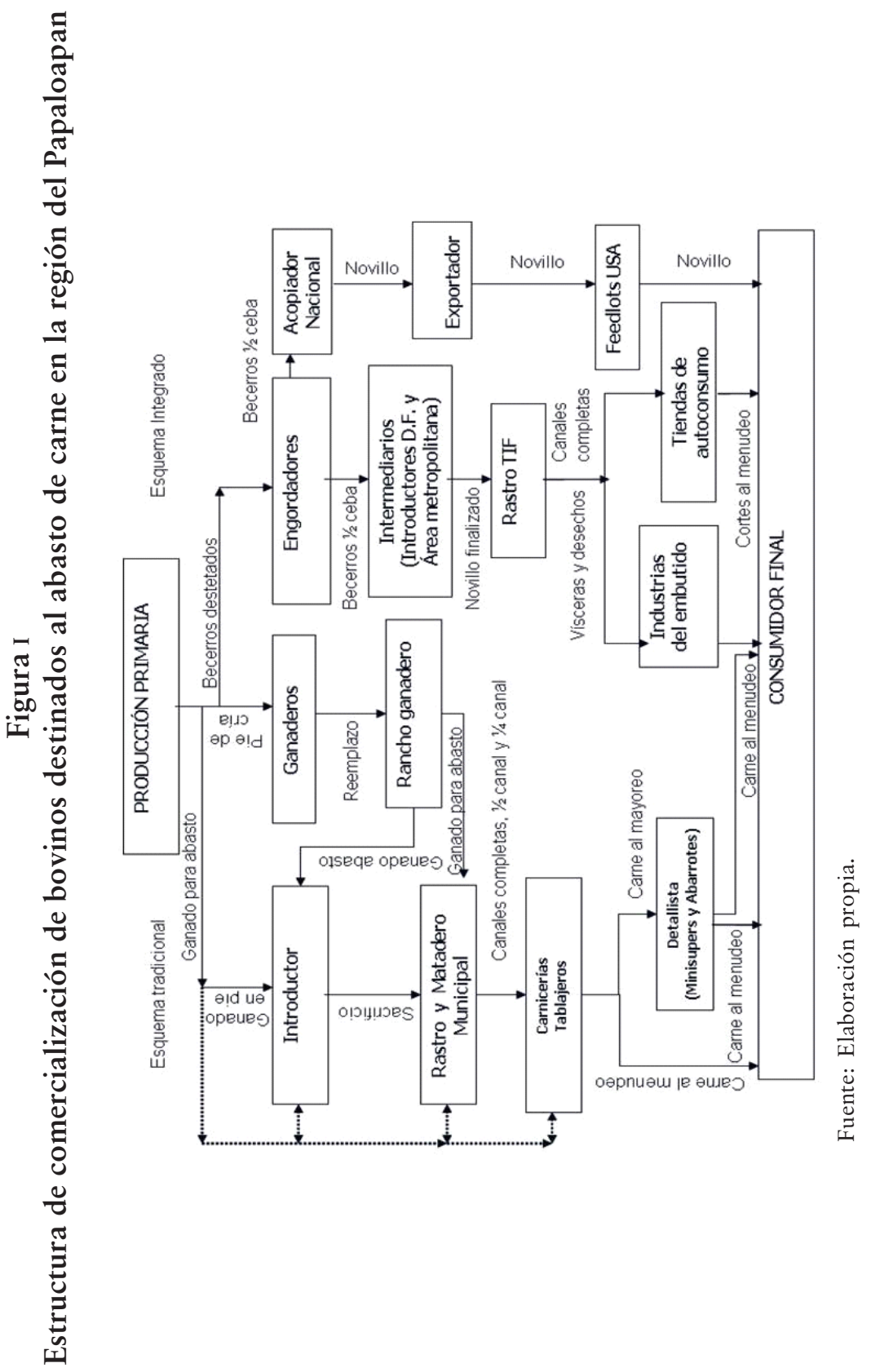


mos, maquinaria y equipo para realizar su función en el canal de comercialización y al darle valor agregado obtener una remuneración por el mismo. Por ejemplo, el productor requiere de terreno, bovinos y tecnología y largos tiempos de proceso, entre otros, para establecer el sistema de producción que determinará la finalidad zootécnica y el enfoque de la producción. El introductor cuenta con el mayor conocimiento del mercado (ubicación de ranchos, precios, tipo de bovino, razas, rendimientos en canal), pero de igual forma requiere de medios de trasporte y capital para realizar la introducción de bovinos a los rastros municipales; no obstante, el tiempo que requiere para hacer su función es breve (de uno a tres días). El rastro o matadero municipal sólo cumple la función de maquila y no interviene directamente en el proceso de comercialización; estos establecimientos requieren de infraestructura adecuada para el sacrificio y obtención de la carne en canal. El tablajero necesita maquinaria y equipo que le permita el despiece de la carne en canal. El consumidor final adquiere la carne de acuerdo con sus gustos y preferencias para satisfacer un requerimiento nutricional.

\section{Caracterización de los agentes participantes}

\subsection{Productores}

De los ganaderos entrevistados, $96 \%$ son hombres y $4 \%$ mujeres. Tienen una escolaridad promedio de $6 \pm 6$ años; el grado máximo de estudios fue licenciatura. La edad fue $53 \pm 13$ años (máxima 90 y mínima 26 años), por lo que son productores maduros. De acuerdo con la teoría, entre más edad tengan se dificulta el cambio en actitud y comportamiento que permita el uso tecnología. Los años de experiencia en la actividad ganadera fue $24 \pm 14$ (intervalo de uno a 79 años); el grado de satisfacción por el desarrollo de la actividad fue muy alto (57\%) y alto (23\%), sólo 20\% mencionó una satisfacción de media a baja. El 81\% de los ganaderos pertenece a la Asociación Ganadera Local (AGL) de su municipio o de otro municipio circunvecino; de este porcentaje, 79\% son hombres y 3\% mujeres; el resto (19\%) no pertenece a la AGL; de este porcentaje, $18 \%$ son hombres y $1 \%$ mujeres. No obstante, tanto socios como no socios realizan la facturación de bovinos (engorda, cría o abasto) en las AGL conforme lo establece la ley vigente (Gobierno del Estado de Veracruz, 1992). Un $60 \%$ (113) de los ganaderos depende económicamente en su to- 
talidad de esta actividad, 11\% (21) entre 50\% y 90\%, 24\% (45) en 50\%, y 4\% (8) en menos de 50\%; además, se encontró que $40 \%$ de los ganaderos tienen sistemas de producción diversificados con cultivos como caña, maíz, frijol, piña, según el municipio donde se encuentren ubicados; esta es una forma de diversificación del riesgo y los protege contra altibajos de mercado y precios, comportamiento típico de la teoría del campesinado.

La heterogeneidad de los productores es alta; los hatos van de 6 a 356 cabezas (promedio 50 bovinos) por productor. Además, se encontraron siete patrones raciales (cuadro 2); de los cuales la cruza suizo con cebú es la más representativa (75.4\%), aspecto que es importante considerar porque conforme se incrementa el porcentaje de Bos indicus en las cruzas, disminuye el peso de la canal y las características de la carne, como el marmoleo; mientras que las cruzas de Bos taurus tienen mayor rendimiento de la carne en canal y mejores características de la misma (Nuñez et al., 2005: 225), al igual que el manejo antes y después del sacrificio del bovino y la faena de la canal de carne (Depetris y Santini, 2000: 2). En la región del Papaloapan se ha documentado que la finalidad zootécnica tiene un doble propósito (86.9\%): pastoreo extensivo y libre pastoreo (98\%) como el sistema predominante, y un patrón racial de la cruza suizo con cebú $(79.8 \%)$ como el más representativo (Vilaboa et al., 2009: 57). Por su escaso nivel tecnológico, este sistema tiende a sobrevivir en periodos especiales de precios altos de los insumos, como la crisis energética y agroalimentaria de 2008 (Espinosa et al., 2000: 651; Pech et al., 2002: 187; Pérez et al., 2003: 96).

Los bovinos destinados al abasto son vacas (91.4\%), vacas y sementales $(1 . \%)$, vacas y novillonas $(5.4 \%)$, vacas y novillos $(1.6 \%)$ y novillos $(0.5 \%)$, datos que concuerdan con lo reportado por Meléndez (2001), Bravo et al. (2002) y Trueta (2003). Este tipo de bovino, destinado al abasto local (municipal) y regional, en otros países se consideran desechos y no aptos para consumo humano; por lo que la calidad de la carne en el esquema tradicional difiere con la del esquema integrado. Sin embargo, al existir la demanda, ya sea por cuestiones económicas o culturales, indica que el consumidor está dispuesto a pagar por ella.

Además de que existen cinco canales de comercialización, el productor distribuye principalmente al tablajero (59\%) y al introductor (35\%); poco más de $6 \%$ de los productores tiene capacidad para introducir bovinos al rastro municipal, ya que cuentan con carnicería propia. En este sentido, Rodas-González (2005: 


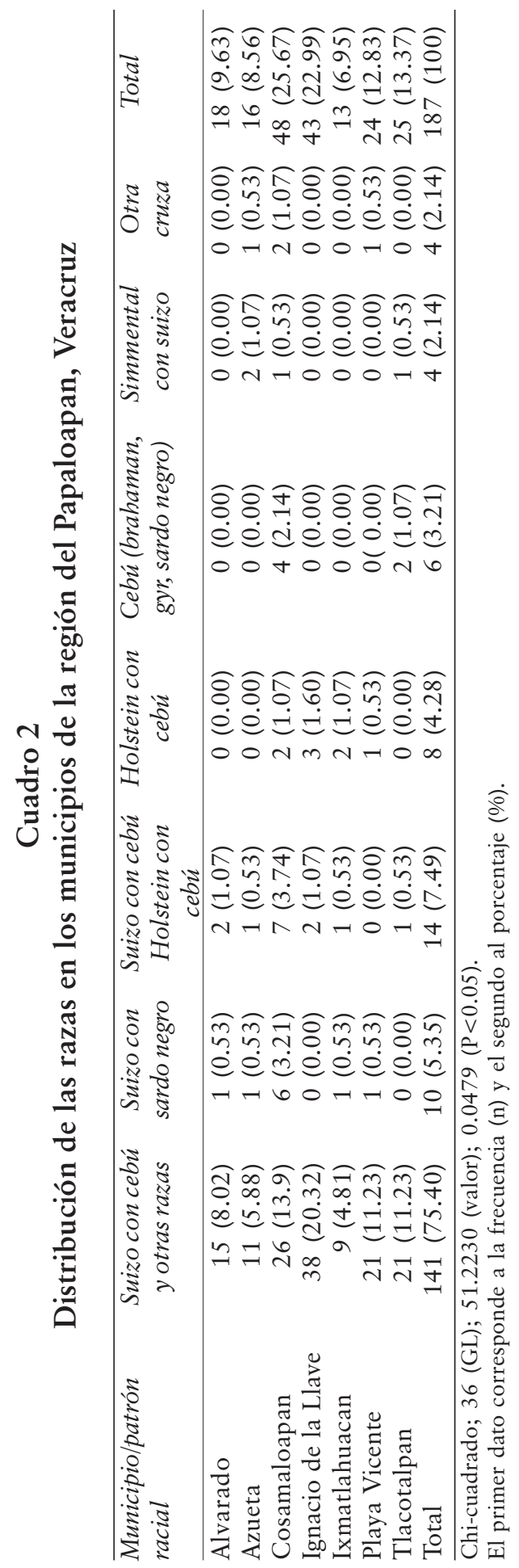


654) menciona que la venta a puerta de corral de grupos heterogéneos de bovinos en clase y peso, reduce el poder de negociación del productor y, por consiguiente, su capacidad para influir en el precio de compraventa. Para los productores, la comercialización es uno de los problemas importantes que deben resolver, ya que dependen en gran medida de la intermediación para colocar la carne en el mercado, porque venden individualmente. La capacidad del productor para vender carne directamente al consumidor, demuestra que es posible reducir los canales de comercialización, lo cual daría mayor competitividad. Sin embargo, el reto es la organización de los productores para comercializar; para ello, se podría aprovechar el esquema jurídico y organizativo de las AGL, que dentro de sus objetivos y funciones prevé la organización de los productores para la comercialización. No obstante, en la actualidad no están desempeñando el papel organizativo y económico que les corresponde. Este agente presenta limitaciones estructurales, derivadas tanto de la limitada escolaridad como de la edad de los productores, asociadas con un reducido tamaño de las explotaciones y una amplia dispersión en el territorio (Caldentey y De Haro, 2004: 24), lo que complica implementar un proceso de integración (Mahecha et al., 2002: 216).

\subsection{Introductores}

Se identificaron 27 introductores de ganado bovino a los rastros municipales, pero su distribución en la región no es uniforme, ya que sólo se encontraron en los rastros de Alvarado (14), Cosamaloapan (10) e Ignacio de la Llave (3). En los municipios de Playa Vicente, Azueta, Ixmatlahuacan y Tlacotalpan la introducción y matanza de bovinos la realizan productores y tablajeros, debido al limitado volumen de matanza anual ( $<300$ bovinos) en los rastros mencionados.

La totalidad de los introductores son hombres, y 93\% de ellos accedió a la entrevista. La edad fue $45 \pm 10$ años (máxima 64 y mínima 26 años); la escolaridad (8 \pm 5 años) fue mayor que en el caso de los productores, que los ubica en un segmento comercial más rentable. La antigüedad en la introducción de bovinos a los rastros municipales es $14 \pm 11$ años (máximo 40 y mínimo 1 año); el grado de satisfacción por esta actividad fue muy alta y alta (48\% y $40 \%$, respectivamente). 
El tipo bovino y razas que adquieren los introductores para sacrificar en el rastro son: $32 \%$ vacas y novillotas; $16 \%$ sólo vacas; $16 \%$ vacas, novillos, novillonas y sementales; $12 \%$ vaca, novillona, novillo; y $8 \%$ vacas y sementales. Los patrones raciales, declarados por los introductores, fueron suizo con cebú (48\%), simmental con cebú y suizo con cebú (32\%); simmental con cebú, suizo con cebú y holstein con cebú (20\%).

El origen de los bovinos es local y de municipios circunvecinos; todos los introductores tienen sus propios proveedores de ganado, así como sus clientes (tablajeros) y no están organizados en ninguna asociación, existe mucha competencia entre ellos y reglas no escritas. El destino de las canales sólo es local o para municipios circunvecinos; se vende como canal completa (44\%), media canal (28\%), y cuartos delantero y trasero (28\%). El 30\% de los introductores tiene carnicerías propias donde venden directamente al público.

La función del introductor es recolectar, comprar y llevar el bovino en pie al rastro municipal para que se convierta en carne en canal para su posterior distribución en las carnicerías locales; para ello, cuenta de manera empírica con una red de información de los productores y la ubicación de los ranchos ganaderos. Asimismo, este agente tiene solvencia y dispone de dinero en efectivo para esperar a que el productor destine bovinos al mercado; además concede crédito a comisionistas y tablajeros para adquirir carne en canal con el compromiso de pago en un periodo determinado. En este sentido, actúa como intermediario financiero y mediante esta acción obtiene ganancias significativas. Sin embargo, no existen contratos legales de compra-venta ni compromisos de ningún tipo más allá de la amistad y tratos verbales. Estos factores, junto con la experiencia y la escolaridad, son los que confieren al introductor la capacidad de realizar prácticas desleales en la comercialización, al tener un mayor control en cuanto a los precios del mercado. No obstante, dicho agente cumple un papel necesario para el funcionamiento del esquema; pero en el caso del esquema integrado, es el primer agente que desaparece.

\subsection{Rastros y mataderos municipales}

De acuerdo con la normatividad de la Dirección General de Ganadería del Gobierno del Estado de Veracruz (DGG), se identi- 
ficaron tres rastros municipales: Alvarado, Ignacio de la Llave y Cosamaloapan. Aunque en este último no existen instalaciones para el sacrifico de bovinos, todo el ganado que se consume allí y en varios municipios circunvecinos (Chacaltianguis, Amatlán, Amatitlán, Tlacotalpan, entre otros), es sacrificado en el Rastro Municipal Modelo ubicado en la cabecera municipal de Carlos A. Carrillo (distante no más de $3 \mathrm{~km}$ de Cosamaloapan). Los mataderos se consideran como tales, básicamente porque no cuentan con la infraestructura sanitaria mínima que debe tener un rastro. En el caso de Ignacio de la Llave, la clasificación oficial la establece como rastro, pero en la práctica funciona como un matadero. La antigüedad de estos establecimientos (rastros y mataderos) es $51 \pm 10$ años, siendo el más antiguo el matadero municipal de Ixmatlahuacan y el más reciente el de Tlacotalpan. Ixmatlahuacan presentó el menor nivel de operación, con un promedio menor a un bovino sacrificado por día. Los mataderos de Azueta y Tlacotalpan tienen un nivel medio de operación con 1.4 y 1.6 bovinos por día; Ignacio de la Llave y Playa Vicente, muestran un nivel alto de operación con 2.4 y 4 bovinos por día. En los rastros de Cosamaloapan (Carlos A. Carrillo) y Alvarado se sacrifican, en promedio, 13 y 20 bovinos por día, respectivamente (cuadro 3) (Sedarpa, 2007). Las vacas son el tipo de ganado que se sacrifica con mayor frecuencia $(91.4 \%)$ y la cruza suizo con cebú $(75.4 \%)$, la más representativa.

El origen del bovino a sacrificar es del municipio donde se ubica el rastro o matadero municipal y de municipios circunvecinos. Sólo en Alvarado se sacrifican novillos con mayor volumen y frecuencia, ya que el mercado destino es la ciudad de Veracruz; en los demás casos el destino de la carne es local y municipios circunvecinos. La cuota en los rastros por el uso de las instalaciones es de $70 \pm 20$ pesos por bovino sacrificado. Los rastros no compran ni venden productos, su única función es maquilar la canal y dar el servicio de sacrificio y destazado. Aunque los gobiernos estatales y municipales proveen la infraestructura, se beneficia más a la intermediación que a los productores. No obstante, puede ser un instrumento de control de varios factores para optimizar el proceso de comercialización.

La problemática identificada en estos establecimientos es de orden operativo, sanitario y de infraestructura; además de problemas no propios de los establecimientos pero relacionados con el mismo, como el transporte de las canales. Los rastros con mayor tecnificación son Alvarado y Carlos A. Carrillo. En los demás 


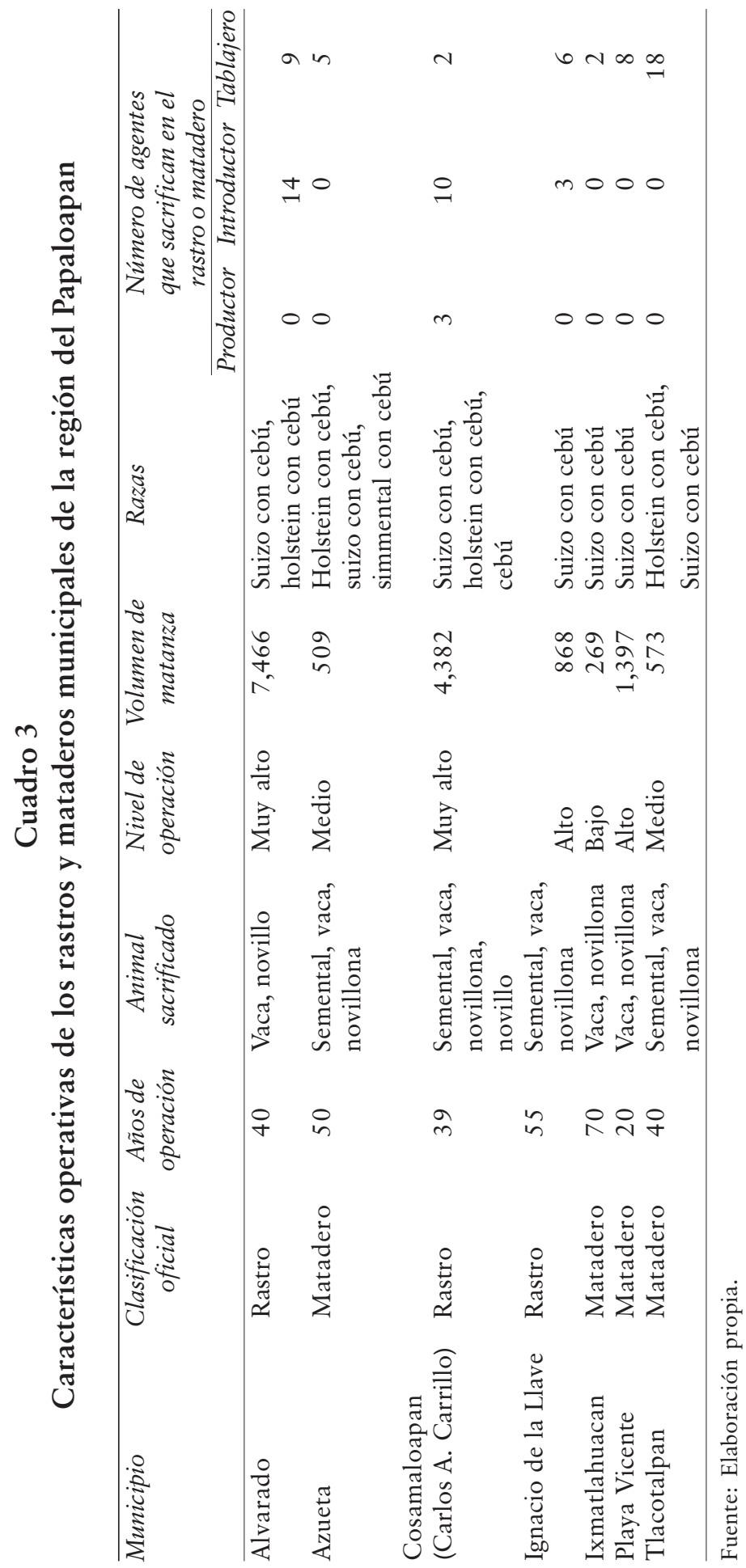


municipios estudiados, la mayoría del sacrificio se realiza de manera rústica (en el piso) y los operadores carecen de la vestimenta y equipo apropiados para desempeñar su labor.

Las carencias de infraestructura, que se encontraron en los establecimientos, son falta de cadenas winchers para mayor sanidad en la matanza y reducir el tiempo de proceso, bombas de agua, alumbrado, sistemas de manejo de desechos y mantenimiento general de las instalaciones; que en el caso de Ixmatlahuacan es obsoleto en su totalidad. Aunado a ello, se encontró el sacrificio de vacas en etapa de gestación. Ninguno de los establecimientos estudiados cumple la normatividad (NOM-008-ZOO1994, NOM-009-ZOO-1994, NOM-033-ZOO-1995, NOM194-SSA1-2004, entre otras) existente, por lo que si se pensara tener acceso a otros mercados (nacionales o internacionales), tendría que haber un proceso de modernización y cambio tecnológico (cadena de frío, sacrificio humanitario, transporte adecuado) en el sistema que permitan un mejor manejo sanitario de la carne. La escolaridad de los administradores es $8.6 \pm 2.7$ años (máximo preparatoria y mínimo primaria) y con una edad promedio de 54 58.8 años (máximo 25 y mínimo 1 año).

\subsection{Tablajeros y detallistas}

El 73\% de los tablajeros, ubicados dentro y fuera de los mercados municipales, no se encuentran organizados como gremio. Un $88.3 \%$ son hombres y $11.7 \%$ mujeres; la edad promedio fue $45 \pm 13$ (máxima 79 y mínima 19 años); la escolaridad $6 \pm 4$ años y la experiencia en la venta de carne 18.8 \pm 14 (52 máximo y mínimo un año); el grado de satisfacción por la actividad fue muy alto (69\%) y alto (14\%). En lo económico, 69\% de los tablajeros dependen totalmente de la actividad, $14 \%$ entre $50 \%$ y $75 \%$ y $17 \%$ depende en $25 \%$ o menos de la actividad. Cerca de $80 \%$ de los bovinos o canales adquiridas provienen de vacas y novillonas. El 58.4\% de los tablajeros adquieren los bovinos en pie para su sacrificio, 34\% compra carne en canal (completa, media y cuartos) al introductor, ya sea en el mismo municipio o en municipios circunvecinos; no obstante, la mitad de los tablajeros que adquieren las canales desconoce de qué raza procedió la canal.

Este estudio permitió identificar un agente de la cadena que no había sido previsto al inicio de este trabajo: el detallista. Este componente ( $8 \%$ del total de tablajeros), identificado en el municipio de Alvarado y conformado en su mayoría por mujeres 
(67\%), adquiere carne al mayoreo en carnicerías de su municipio dentro o fuera del mercado municipal para vender la carne al detalle. Una característica importante en este agente radica en que funcionan como tiendas de abarrotes o minisupers, en pequeñas localidades que no cuentan con carnicerías establecidas; donde no es factible la matanza y consumo de un bovino al día. La venta de carne no es la finalidad primordial del negocio, sino que sirve de atracción para que los consumidores al ir a comprar otros productos adquieran la carne o viceversa. El detallista aumenta un eslabón más al canal de comercialización que se refleja en un incremento en el precio al consumidor final. Este agente se favorece por el descuento al mayoreo proporcionado por el tablajero y por el incremento en el precio ofrecido al consumidor final. El destino de la carne es netamente local, sobre todo para consumo familiar, la cual adquiere el público en general (51\%) y amas de casa (42\%), y en menor medida pequeños restaurantes (fondas) y otros negocios de los municipios donde se encuentran instalados (7\%). Otro aspecto que es importante destacar es que algunas carnicerías surten a las taquerías locales, carne que los tablajeros consideran de segunda calidad, la cual dan a menor precio que al público en general. Existe un gran número de tablajeros como para asumir que existe competencia en el ámbito regional; sin embargo, pueden actuar monopólicamente en comunidades donde sólo existe una carnicería. Aquí también hay ineficiencia del mercado porque no existe homogeneidad en la calidad y precio de la carne al consumidor. Sin embargo, al haber necesidad de alimentos baratos, asociados a una baja calidad, permiten que las prácticas actuales de este agente continúen hasta que el consumidor sea más exigente. Este agente aporta el mayor valor agregado al producto por la diversificación de cortes que destina al consumidor.

\subsection{Consumidores}

En México, según una encuesta de la Sagarpa (2004: 13), del total de hogares que gastan un porcentaje de su ingreso en la compra de carnes, aproximadamente $72 \%$ destina una proporción a la compra de carne bovina, la cual oscila entre 10 y 15\%; siendo los cortes más consumidos el bistec y la milanesa, y en orden descendente pulpa, costilla y carne molida; a los cortes como lomo, filete y cortes especiales se les destina un menor porcentaje del ingreso, datos que coinciden con los encontrados en 
la región del Papaloapan, donde 40\% de los consumidores entrevistados fueron hombres y $60 \%$ mujeres. La mayoría de los entrevistados se dedican a labores domésticas (36\%), asalariados (21\%) y propietarios de negocio (21\%), profesionistas $(8 \%)$ y actividades agropecuarios (4\%), además, 9.8\% no especificaron actividad laboral; datos que concuerdan con Meléndez (2001: 244) y Segovia y Albornoz (2005: 673), quienes mencionan que el consumidor de carne se compone sobre todo de amas de casa y público en general de todos los estratos sociales. La edad fue $44 \pm 14$ años (89 máximo y mínimo 16 años); la escolaridad de $8 \pm 5$ años; las familias se integran por $4 \pm 2$ miembros. La preferencia por el consumo de carne de res es de media (42\%) a alta (25\%); sólo 6\% manifestó una preferencia muy alta por el consumo de carne bovina. La frecuencia de consumo es de una (32\%) a tres veces (20\%) por semana. Los lugares de compra son carnicerías establecidas fuera de los mercados municipales (58\%), mercados municipales (37\%), supermercados y tiendas de abarrotes (4\%), así como tiendas de autoservicio (1\%). En las comunidades semiurbanas y rurales los consumidores son menos exigentes en cuanto a calidad e higiene de la carne debido a la escasez en los ingresos. En general, el consumidor cuenta con poco conocimiento respecto a las características del producto y ejerce limitadamente su poder de elección, ya que consume la carne que se ofrece en el mercado.

\section{Conclusiones e implicaciones}

El esquema de comercialización tradicional (no integrado) de bovinos destinados para el abasto de carne en la región del Papaloapan, enfocado al abasto interno, está conformado por los agentes: productor, introductor, rastro municipal, tablajero, detallista y consumidor. Estos agentes se interrelacionan entre sí, en diversas combinaciones, para establecer los canales de distribución de la carne, por lo que en las condiciones actuales, su presencia es necesaria para que dicho esquema funcie.

El productor tiene limitaciones estructurales relacionadas con aspectos socioeconómicos y productivos, lo cual complica implementar procesos de organización e integración para la comercialización. Los agentes intermedios participan con mejores condiciones tanto económicas como sociales, mientras que el consumidor final presenta un limitado poder de elección. Esta industria tiene potencial para ser competitiva internacionalmen- 
te, pero el procesamiento y la comercialización son obstáculos que se deben sortear para lograrlo.

\section{Agradecimientos}

Queremos agradecer a todos los productores, introductores, administradores de rastros, tablajeros y consumidores de la región del Papaloapan, quienes amablemente participaron en las entrevistas. A las autoridades municipales por las facilidades otorgadas para la realización de este trabajo. Al Consejo Nacional de Ciencia y Tecnología (Conacyt) por la beca otorgada (2006-2009). Al Colegio de Postgraduados, Campus Veracruz, por el apoyo y las facilidades autorizadas.

\section{Bibliografía}

Bernanke, Ben y Robert Frank (2007), “Oferta y demanda”, en Tercera Edición, Principios de economía, McGraw Hill, México, pp. 62-90.

Bravo, Francisco Javier, Roberto García, Gustavo García y Enrique López (2002), "Márgenes de comercialización de la carne de res proveniente de la cuenca del Papaloapan, en el mercado de la ciudad de México”, Agrociencia, 36 (2), México, pp. 255-266.

Caldentey, Albert Pedro (1986), Comercialización de productos agrarios. Aspectos económicos y comerciales. Agrícola Española, Tercera Edición. España pp. 19-21

Caldentey, Albert Pedro y Tomás de Haro (2004), "Agentes y canales de comercialización", en Comercialización de productos agrarios, Quinta Edición. Agrícola Española, España, pp. 21-37.

Depetris, Gustavo y Francisco Santini (2000), "Calidad de carne asociada al sistema de producción”, Grupo de Nutrición, Metabolismo y Calidad de Producto, Jornadas Internacionales en Carnes Vacunas, Instituto Nacional de Tecnología Agropecuaria (INTA), Estación Experimental Balcarce, Argentina. 
Espinosa, José, Jaime Matus-Gardea, Miguel Ángel Martínez, María Santiago, Eriberto Román y Lauro Bucio (2000), "Análisis económico de la tecnología bovina de doble propósito en Tabasco y Veracruz", Agrociencia, 34 (5), México, pp. 651-661.

García-Mata, Roberto, Ángel García y Roberto García (2003), Teoría del mercado de productos agropecuarios, Colegio de Postgraduados, Texcoco, México, pp. 143-215.

Gobierno del Estado de Veracruz (2005), Programa Veracruzano de Agricultura, Ganadería, Forestal, Pesca y Alimentación 2005-2010, Sedarpa, www.secver.gob.mx/difusion/ pvd/PVD2005-2010.pdf, diciembre de 2008.

Gobierno del Estado de Veracruz (1992), Ley ganadera para el estado de Veracruz, http://www.ordenjuridico.gob.mx/Estatal/VERACRUZ/Leyes/VERLEY67.pdf.

Gomes de Cam, Valle, Mario (1999), Prospección de cadenas agroalimentarias, Empresa Brasilera de Pesquisa Agropecuaria-IICA-Procitrópico, Venezuela.

Huerta, N. (2005), "Puntos críticos antes y después del beneficio para asegurar la calidad de la carne", en Carlos González-Stagnaro y Eleazar Soto (eds.), Manual de ganadería doble propósito, Fundación Girarz, Venezuela, p. 4.

INEGI (Instituto Nacional de Geografía, Estadística e Informática), Censo Agropecuario 2005, www.inegi.gob.mx.

Mahecha, Liliana, Luis Gallego y Francisco Peláez (2002), "Situación actual de la ganadería de carne en Colombia y alternativas para impulsar su competitividad y sostenibilidad", Revista Colombiana Ciencia Pecuaria, 15 (2), Universidad de Antioquía, Antioquía, pp. 213-225.

Márquez, Isacc, Roberto García-Mata, Gustavo García, José Saturnino y Enrique López (2004), "El efecto de las importaciones de carne bovina en el mercado interno mexicano, 1991-2001”, Agrociencia (38), México, pp. 121-130. 
Meléndez, Ricardo (2001), "Mercadotecnia”, en Administración pecuaria (bovinos), Universidad Nacional Autónoma de México, México, pp. 203-255.

Nuñez, Francisco, Alfredo García, José Arturo García, Jorge Hernández y Jorge Alfonso Jiménez (2005), "Caracterización de canales de ganado bovino en los valles centrales de Oaxaca", Técnica Pecuaria en México, 43 (2), México, pp. 219-228.

Pech, Víctor, Jorge Santos y Rubén Montes (2002), “Función de producción de la ganadería de doble propósito en la zona oriente del estado de Yucatán, México", Técnica Pecuaria en México, 40, México, 187-192.

Pérez, P., Rolando Rojo, María del Carmen Álvarez, José Juan García, Catarino Ávila, Silvia López, Juan Antonio Villanueva, Héctor Chalate, Eusebio Ortega y Jaime Gallegos (2003), Necesidades, investigación y transferencia de tecnología de la cadena de bovinos de doble propósito en el estado de Veracruz, Fundación Produce Veracruz, México.

Rodas-González, Argenis (2005), "Limitantes y vicios del sistema de categorización venezolana de canales bovinas", en C. González-Stagnaro y E. Soto Belloso (eds.), Manual de ganadería doble propósito, Fundación Girarz, Maracaibo, pp. 654-659.

Ruiz, Agustín, Leticia Míriam Sagarraga, José María Salas, Heriberto Estrella, Agustín Ruíz, Mariano González y Ángel Juárez (2004), Impacto del TLC en la cadena de valor de bovinos para carne, Universidad Autónoma Chapingo, México.

Sagarpa (Secretaría de Agricultura, Ganadería, Desarrollo Rural, Pesca y Alimentación) (2004), Situación actual y perspectivas de la producción de carne bovino en México", http:/ /www.ipcva.com.ar/files/mexico.pdf.

Sagarpa (Secretaría de Agricultura, Ganadería, Desarrollo Rural, Pesca y Alimentación) (2006), Situación actual y perspec- 
tivas de la carne bovino en México", http://www.sagarpa. gob.mx/Dgg/estudio/sitbov06.pdf.

Sagarpa-SIAP (Secretaría de Agricultura, Ganadería, Desarrollo Rural, Pesca y Alimentación-Sistema de Información y Estadística Agropecuaria y Pesquera (2005), "Estadística básica. Estadísticas del sector ganadero. Población ganadera 1996-2005 (carne y leche)", Sagarpa-SIAP, http:// www.siap.sagarpa.gob.mx/, marzo de 2008.

Sedarpa (Secretaría de Desarrollo Agropecuario, Rural, Forestal y Pesca) (2007), Inventario ganadero y sacrificio de ganado (base de datos años 2005-2006), Dirección General de Ganadería-Gobierno del Estado de Veracruz, Jalapa.

Segovia, Emma Arlenis Albornoz (2005), "Importancia de las carnicerías tradicionales en el circuito cárnico", en C. González-Stagnaro y E. Soto Belloso (eds.), Manual de ganadería doble propósito, Fundación Girarz, Maracaibo, pp. 671-675.

Tansini, Rubén (ed.) (2003), “La economía como ciencia social”, en Economía para no economistas, Universidad de la República, Montevideo, http://decon.edu.uy/publica/noec/.

Trueta, Rafael (2003), "Crónica de una muerte anunciada. Impacto del TLC en la ganadería bovina mexicana", Memorias, XVII Congreso Nacional de Buiatría, Asociación Mexicana de Médicos Especialistas en Bovinos (AmmeB), Villahermosa, Tabasco, México, pp. 57-87.

Vilaboa, Julio, Pablo Díaz, Octavio Ruiz, Diego Platas, Sergio González y Francisco Juárez (2009), “Caracterización socioeconómica y tecnológica de los agroecosistemas con bovinos de doble propósito en la región del Papaloapan, Veracruz", Tropical and Subtropical Agroecosystem, 10 (1), Universidad Autónoma de Yucatán, Mérida, pp.53-62.

Recibido: 2 de mayo de 2008. Reenviado: 17 de febrero de 2009. Aceptado: 5 de marzo de 2009. 
Julio Vilaboa Arroniz. Es maestro en ciencias por el Colegio de Postgraduados, Campus Veracruz; es ingeniero agrónomo por el Instituto Tecnológico Agropecuario núm. 3 de Tuxtepec, Oaxaca; y Diplomado en la Unidad de Capacitación para el Desarrollo Rural (Uncader). Actualmente es candidato a doctor en ciencias en el Programa de Agroecosistemas Tropicales del Colegio de Postgraduados, Campus Veracruz. Sus líneas de investigación son: área de socioeconomía y ganadería tropical. Entre sus publicaciones destacan: "Productividad y autonomía en dos sistemas de producción ovina: dos propiedades emergentes de los agroecosistemas", Interciencia, 31 (1), Caracas, pp. 37-44 (2006); "Conformación corporal de las razas ovinas pelibuey, kathadin y doper en el estado de Veracruz", Asociación Mexicana de Técnicos Especialistas en Ovinos (AMTEO), Gaceta, 3, AMTEO, 14 p. (2007); "Caracterización socioeconómica y tecnológica de los agroecosistemas con bovinos de doble propósito en la región del Papaloapan, Veracruz", Tropical and Subtropical Agroecosystem, 10 (1), Universidad Autónoma de Yucatán, Mérida, pp. 53-62 (2009).

Pablo Díaz Rivera. Es doctor por la Universidad de Florencia, Italia; hizo la maestría en el Programa de Ganadería del Colegio de Postgraduados, Campus Montecillo, Texcoco; y la licenciatura en el Departamento de Zootecnia de la Universidad Autónoma Chapingo. Actualmente es profesor-investigador asociado en el Colegio de Postgraduados, Campus Veracruz. Es candidato en el Sistema Nacional de Investigadores (SNI). Sus líneas de investigación son: sistemas de producción animal tropical y conservación de recursos genéticos animales. Entre sus publicaciones destacan: "Ganadería bovina de doble propósito: problemática y perspectivas hacia un desarrollo sustentable”, en C. González Stagnaro y E. Soto Belloso (eds.), Desarrollo sostenible de la ganadería doble propósito, Fundación Girarz, Maracaibo, pp. 5869 (2008); "Resistencia a parásitos gastrointestinales en ovinos florida, pelibuey y sus cruzas en el trópico mexicano", Agrociencia, 34 (1), México, pp. 13-20; "Caracterización morfológica y genética de las razas ovinas toscanas pomarancina y garfagnina blanca consideradas en peligro de extinción", Memorias, XVI Reunión Científica Tecnológica Forestal y Agropecuaria Veracruz2003, 6 y 7 de noviembre, Veracruz. 
Diego Esteban Platas Rosado. Es doctor por la Universidad de Minnesota; hizo la maestría y la licenciatura en la Universidad Autónoma Chapingo. Actualmente es profesor-investigador asociado en el Colegio de Postgraduados, Campus Veracruz. Sus líneas de investigación son: agronegocios y economía de los recursos naturales y medio ambiente, y sostenibilidad de agroecosistemas tropicales. Entre sus publicaciones destacan: "Evaluating Economic Impacts of an Evolving Swine Industry: The Importance of Region Size", Review of Agricultural Economics, 24 (2), Agricultural and Applied Economical Association, Arizona, pp. 458-473 (2002); “IMPLAN's weakest link: production functions or regional purchase coefficients", Journal of Regional Analysis and Policy, 32 (1), Continent Regional Science Association, Kansas, pp. 33-48 (2002).

Octavio Ruiz Rosado. Es doctor por el Colegio Imperial de la Universidad de Londres; hizo la maestría en la Universidad de California, Santa Cruz; y la licenciatura en el Colegio Superior de Agricultura Tropical, en Tabasco. Actualmente es profesor-investigador asociado en el Colegio de Postgraduados, Campus Veracruz. Sus líneas de investigación son: estudios agroecológicos, socioeconómicos y de recursos naturales, así como transferencia de tecnología. Entre sus publicaciones destacan: "The systems approach for sustainable development at catchment and parish group levels", International Journal of Sustainable Development and World Ecology, 8, Sapiens Publishing, pp. $79-84$ (2001); "Agricultural sustainability in Kent, England: the systems approach at watershed level a strategy for its development", en Proceedings of the 1999 International Sustainable Development Research Conference, Universidad de Leeds, Reino Unido, pp. 317-322 (2000); “Cambios en propiedades del suelo de un agroecosistema citrícola inducidos por la época del año, edad de árboles y pastoreo de ovinos”, Agrociencia, 33, México, pp. 149-158 (1999).

Sergio Segundo González Muñoz. Es doctor por la Universidad de Lincoln, Nebraska; hizo la maestría en esta misma institución y la licenciatura en la Universidad de Kingsville, Texas. Sus líneas de investigación son: nutrición animal y ganadería. Actualmente es investigador nivel III en el Sistema Nacional de Investigadores (SNI) y es profesor investigador titular en el Programa de Ganadería del Colegio de Postgraduados. Entre sus publicaciones destacan: "The effect of plant age on the chemical composi- 
tion of fresh and ensiled Agave salmiana leaves", South African Journal of Animal Science, 38 (1) SASSAS, pp. 43-50 (2008); "Effects of exogenous fibrolytic enzymes on ruminal fermentation and digestibility of total mixed rations fed to lambs", Animal Feed Science and Technology, 142, ELSERVIER, pp. 210-219 (2007); "Efecto de enzimas fibrolíticas exógenas en la degradación ruminal in vitro de dietas para vacas lecheras", Interciencia, 32 (12), Caracas, pp. 850-853 (2005).

Francisco Juárez Lagunes. Es doctor por la Universidad de Cornell, Nueva York; hizo la maestría en ciencias en la Facultad de Medicina Veterinaria y Zootecnia de la Universidad Veracruzana; y la licenciatura en la Universidad Nacional Autónoma de México. Actualmente es investigador de tiempo completo en la Facultad de Medicina, Veterinaria y Zootecnia de la Universidad Veracruzana. Sus líneas de investigación son: nutrición de rumiantes (bovinos) y ganadería tropical. Entre sus publicaciones destacan: "Evaluation of tropical grasses for milk production by dual-purpose cows in tropical Mexico", Journal of Dairy Science, 82 (10), American Dairy Science Association, pp. 2136-2145 (1999). 